\title{
DIFFERENTIAL SENSOR FOR PH MONITORING OF ENVIRONMENTAL OBJECTS
}

\author{
Sergey Romanenko, Timofey Radenkov, Egor Newsky and Artur Kagirov ${ }^{a}$ \\ Ecology and Life Safety Department, Non-destructive control institute, Tomsk Polytechnic \\ University, 634050, Tomsk, Russia
}

\begin{abstract}
Differential pH sensor is proposed. Reference electrode and measuring electrode are the same type. Reference electrode is immersed in standard buffer solution with known $\mathrm{pH}$ value. The differential $\mathrm{pH}$ sensor has longer service life as compared with the traditionally used sensors with silver chloride reference electrode. Ultrasonic cleaning system is proposed to clean the primary measuring transducer from pollution that form as result of silting during long-term operation with the sensor.
\end{abstract}

\section{Introduction}

Currently, one of the most common methods of environmental monitoring is an online autonomous monitoring. In Russia, the most common monitoring system is sampling and subsequent analysis in the laboratory, and, in rare cases, the mobile monitoring. In turn, the autonomous remote monitoring is a promising step in the development of environmental objects control methods [1-4].

Development of the automated analytical complex for monitoring of key indicators of water quality (after cleaning) and control of the techno logical cleaning process is challenging because it needs selectivity and sensitivity of the determination of the components, their diversity, as well as ensuring a reliable long-term operation of sensors and the preservation of their sensitivity and calibration at an acceptable cost.

The main impediment to the creation of devices for autonomous monitoring, is the lack of transducers providing reliable long-term operation, while maintaining selectivity and sensitivity [5].

$\mathrm{pH}$ control is an integral part of the enviromental objects monitoring, as in many cases, the $\mathrm{pH}$ value is a informative indicator of the state of the environment. Measuring of $\mathrm{pH}$ value is realized in different laboratories, both stationary and mobile, including a those working in the field conditions [6-9]. Potentiometric analysis methods are effective for the control of the environment. These methods are characterized by high sensitivity and selectivity, high speed of response to changes in the composition of the object being analyzed, ease of automation and possibility of remote control. Finally, they do not require expensive analytical equipment and can be used in laboratory, production and field conditions $[10,11]$.

${ }^{\text {a } C o r r e s p o n d i n g ~ a u t h o r: ~ k a g i r o v @ t p u . r u ~}$ 
The aim of the study is to develop a differential sensor for $\mathrm{pH}$ monitoring of environmental objects. The main objectives are:

- procuring of $\mathrm{pH}$ at-site analysis and simplification of sensor calibration procedure;

- ensuring long life (few months) of the sensor without the need for its servicing.

A wide variety of sensors for $\mathrm{pH}$-meters is currently produced $[12,13]$. Most of them take into consideration the dependence of the potential on the temperature, but their use involves the provision of laboratory conditions to run correctly.

Thus, it nesessary a search of $\mathrm{pH}$ sensor for continuous monitoring of environmental objects having indications stability in long time and strength sufficient for use in production and field conditions.

Also, there is the problem of finding the reference electrode having a stable potential. Silver chloride electrode is most widely used today [14]. It is known that the main factor reducing the operating life of $\mathrm{pH}$ sensor is the instability of the reference electrode potential, in particular silver chloride electrode. Its disadvantages are:

- a relatively high rate of electrolyte expiration from electrode;

- a favorable medium for microorganisms growing;

- a dilution of internal solution, which leads to change of the electrode potential caused by change of the internal concentration of the solution.

Thus, for increasing the lifetime of the $\mathrm{pH}$ sensor has been proposed the differential measurement scheme, which avoids the shortcomings of the silver chloride reference electrode.

\section{Main partition}

Differential pH sensor (Figure 1) with the ion-selective electrode comprises a body 1 with a measuring glass electrode 2 and the reference electrode 4 . The measuring glass electrode 2 and the reference electrode 4 are connected with the secondary transducer 5 . The reference electrode 4 is glass electrode 3, immersed in reference solution 6 having a buffer capacity. The electrodes are mounted in the body by sealing elements 7,8 . In order to determine the $\mathrm{pH}$ value, body of sensor with electrodes is placed in the analyzed medium 9.

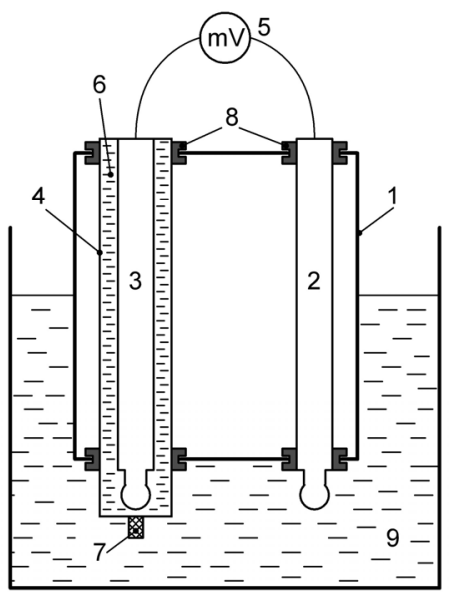

Figure1. Functional scheme of differential $\mathrm{pH}$ sensor with ion-selective glass electrodes.

The sensor works like this: when it is immersed in the analyzed medium on the measuring glass electrode appears the potential, which is proportional to the $\mathrm{pH}$ value. Since the glass electrode 3 is placed in a solution 6 having a stable $\mathrm{pH}$ value, its potential is 
constant. The porous membrane eliminates mixing analyzed medium and a reference solution having a buffer capacity.

The potential of a glass electrode depends linearly on the $\mathrm{pH}$ :

$$
E=E^{\circ}-b \mathrm{pH},
$$

where: $E^{\mathrm{o}}$-the standard potential of the glass electrode; $b=R T \ln 10 / F$-factor depending on the temperature; $\mathrm{pH}-\mathrm{pH}$ value.

Then the potential of the measuring electrode is equal:

$$
E_{1}=E_{1}^{\circ}-b \mathrm{pH}
$$

Reference electrode potential is equal:

$$
E_{2}=E_{2}^{\circ}-b \mathrm{pH}_{\mathrm{ref}}
$$

where $\mathrm{pH}_{\mathrm{ref}}$ - potential of the reference solution.

Since the indicator electrode and the reference electrode are the same type, the $E_{1}^{\circ}$ and $E_{2}^{\circ}$ is also the same. Thus, the potential difference between the indicator electrode and the reference electrode, measured by secondary transducer is determined by the following relation:

$$
E=b\left(\mathrm{pH}_{\mathrm{ref}}-\mathrm{pH}\right) .
$$

Since the solution having a buffer capacity, has a known $\mathrm{pH}$, there is no need to calibrate the electrodes on a series of buffers [15]. As a solution having a buffer capacity, we used phosphate buffer $(\mathrm{pH}=6.86)$, in which 25 -fold dilution causes a change in its $\mathrm{pH}$ not more than $0.05 \mathrm{pH}$-units.

The $b$ coefficient is the only parameter used in the recalculation potential $E$ to $\mathrm{pH}$. The steepness of the calibration curve $b$ depend of temperature, the influence of which can be take into account in secondary transducer.

Calibration of the differential $\mathrm{pH}$ sensor with ion-selective electrodes can be made on one point. For this purpose, the device is immersed in the analyzed medium with a known $\mathrm{pH}$ value and measured value of the potential difference $E$ between the measuring glass electrode and auxiliary reference electrode. Then calculate coefficient $b$ :

$$
b=\frac{E}{\mathrm{pH}_{\mathrm{ref}}-\mathrm{pH}} \text {. }
$$

To improve the accuracy of $\mathrm{b}$ when the device calibration is performed is necessary that the difference in $\mathrm{pH}$ between the sample solution and $\mathrm{pH}_{\text {ref }}$ was maximum. For example, when the internal solution $\mathrm{pH}_{\mathrm{ref}}=6.86$ buffers with 1.65 or $12.43 \mathrm{pH}$ can be used for calibration.

When used as a first transducer glass electrode measuring scheme must satisfy a number of conditions. On the water you need to use operational amplifiers with input impedance more than $10 \mathrm{GOhm}$ and low leakage currents. As the input amplifier was selected AD8606. Simplified measuring scheme of differential pH sensor is shown in Figure 2.

The measuring electrode is connected to the noninverting input of the operational amplifier AD8606. The reference electrode is connected to the output of the operational amplifier, the potential of which relative to the internal ground potential as half the supply voltage. After RC chain with a cutoff frequency of $20 \mathrm{~Hz}$ filtered signal is input to an analog-to-digital converter AD1115. In I2C bus microcontroller receives a digital signal corresponding to the potential difference between the indicator and reference electrode. 


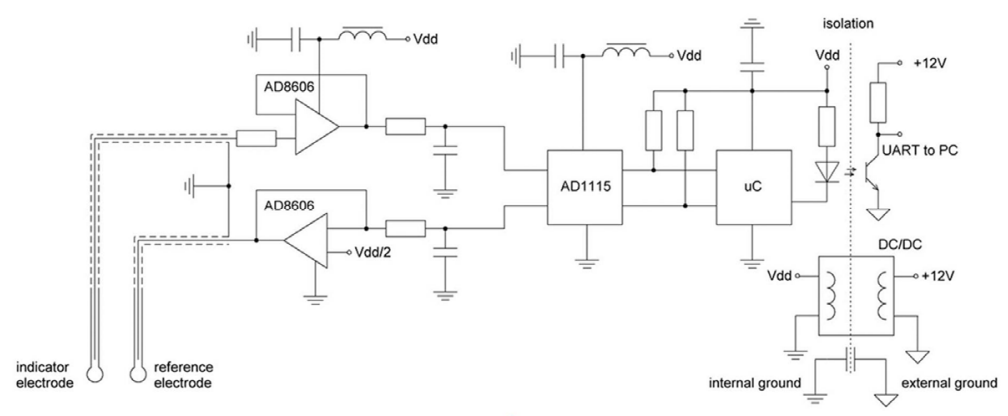

Figure 2. Simplified measuring scheme of differential $\mathrm{pH}$ sensor.

To account for the temperature dependence of the electromotive force sensor has a temperature measurement channel on a digital thermometer DS1820. At measured potential difference and temperature microcontroller calculates $\mathrm{pH}$ from equation

$$
\mathrm{pH}=\mathrm{pH}_{\mathrm{ref}}-\frac{298 E}{b^{\prime} T},
$$

where $b^{\prime}$ - experimentally received slope of calibration curve at $298 \mathrm{~K}$.

The result is transmitted by UART to a computer via photocoupler, which provides galvanic isolation between internal and external ground. Isolating power supply converts the input voltage of $12 \mathrm{~V}$ to output $7 \mathrm{~V}$, then this voltage is supplied to the linear stabilizer 7805 to generate $5 \mathrm{~V}$ voltage for analog and digital circuits $V_{d d}$. To reduce electromagnetic noises internal and external ground are connected by capacitor.

Calibration characteristics of the differential $\mathrm{pH}$ sensor with the new electrodes and after six months of operation are shown in Figure 3.
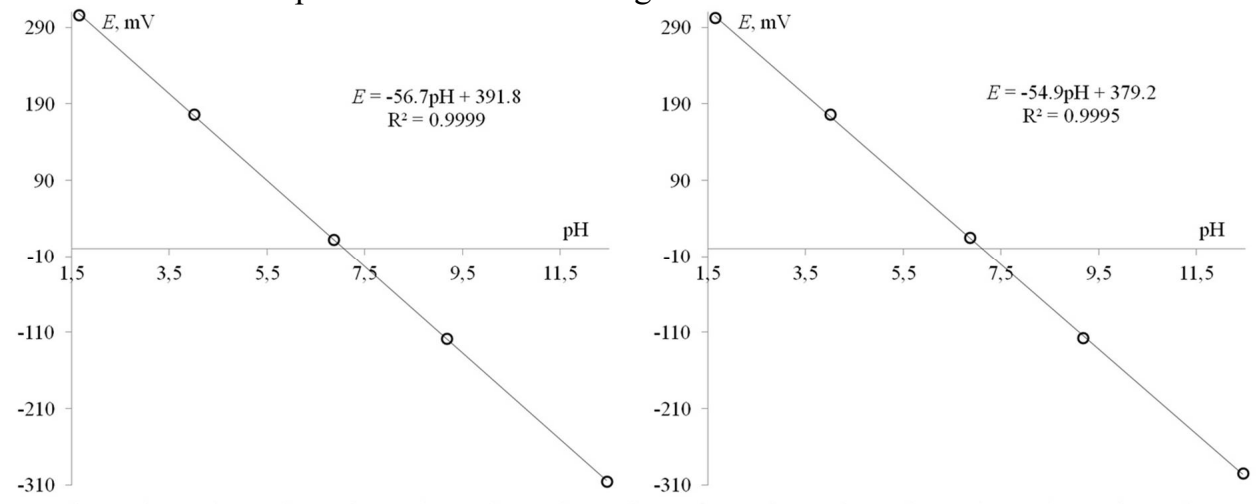

Figure 3. The calibration characteristics of the differential $\mathrm{pH}$ sensor with new ion-selective electrodes (left) and after six months of operation (right).

It should be noted important property of calibration characteristics: only its steepness (b) is changed in continuous operation of the differential sensor, while the isopotential point coordinates remain constant $\mathrm{E}=0, \mathrm{pH}=6.86$.

During prolonged continuous operation in natural water sensor can accrete silt or algae, leading to measurement error. To ensure the purity of the transducers ultrasonic cleaning system was used [16, 17], shown in photos differential sensor (Figure 4). 


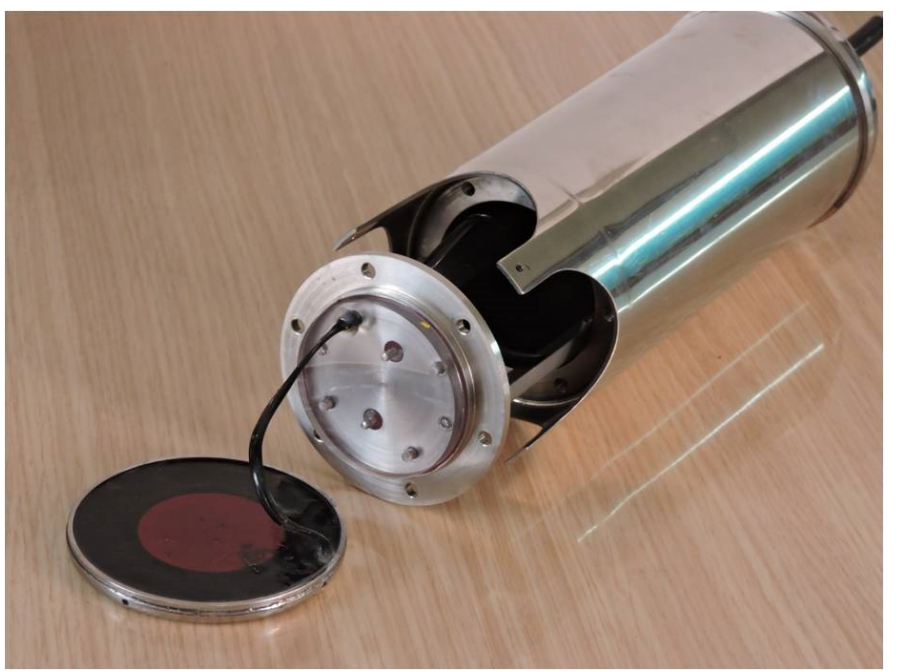

Figure 4. Photo of the differential $\mathrm{pH}$ sensor with ultrasonic cleaning system (bottom left).

Ultrasonic cleaning system is a pulsed boost converter supplying the piezoelectric element $40 \mathrm{~mm}$ in diameter. The microcontroller switches on cleaning system once every five minutes for 20 seconds, this mode allows to maintain a clean transducer even in the most adverse conditions. The power consumed by the sensor during cleaning, is not more than $30 \mathrm{~W}$, and in the measurement mode without purification less than $1 \mathrm{~W}$. The diameter of sensor $100 \mathrm{~mm}$, length $350 \mathrm{~mm}$, weight $1.5 \mathrm{~kg}$.

\section{Conclusion}

Advantages of the proposed design of the sensor, realizing the differential measuring scheme:

— simplifying the calibration procedure (one-point, instead of the traditional twopoint);

- increasing the duration of continuous operation through the use of a glass electrode in phosphate buffer $\mathrm{pH} 6.86$ as the reference electrode;

- ultrasonic cleaning system that supports transducers clean and does not allow the ion-selective electrodes to grow algae or sludge.

During the six months of continuous operation of the sensor the steepness of calibration function has decreased from 56.7 to $54.9 \mathrm{mV} / \mathrm{pH}$, which corresponds to the measurement error in the range $0-12 \mathrm{pH}$, no more than $0.1 \mathrm{pH}$. An important feature of the differential $\mathrm{pH}$ measurement scheme is a long-term stability of the isopotential point $(\mathrm{pH}=6.86)$.

\section{Acknowledgment}

The reported study was supported by RFBR, research project No. 16-48-700230.

\section{References}

[1] P. Jaikang, K. Grudpan, T. Kanyanee, Talanta 132, 884 (2015) doi: $10.1016 /$ j.talanta.2014.10.046

[2] A. Kagirov, S. Romanenko, Testing. Diagnostics (Sp), 157 (2011) 
[3] S.H.A. Hassan, S.W. Van Ginkel, M.A.M. Hussein, R. Abskharon, S.-E. Oh, Environment International 92-93, 106 (2016) doi: 10.1016/j.envint.2016.03.003

[4] A. Kagirov, D. Kalashnikova, Testing. Diagnostics (13), 73 (2014)

[5] B.S. Echols , R.J. Currie, D.S. Cherry, J.R. Voshell, Environmental Monitoring and Assessment 185, 1341 (2013) doi: 10.1007/s10661-012-2636-7

[6] C. Manjarrés, D. Garizado, M. Obregon, N. Socarras, M. Calle, C. JimenezJorquera, Journal of Applied Research and Technology 14, 1 (2016) doi: $10.1016 /$ j.jart.2016.01.003

[7] T. Radenkov, S. Romanenko, A. Kagirov, Testing. Diagnostics (Sp), 146 (2011)

[8] A. Kulasekaran, G. Andal, R. Lakshimipathy, J. John Alexander, International Journal of ChemTech Research 8, 16 (2015)

[9] F. Scholz, T. Steinhardt, H. Kahlert, J. Behnert, Electroanalysis 16, 2058 (2004) doi: 10.1002/elan.200403059

[10] J. Plambeck, Electroanalytical chemistry: basic principles and applications (Wiley, New York, 1982)

[11] M. Sander, T.B. Hofstetter, C.A. Gorski, Environmental Science and Technology 49, 5862 (2015) doi: 10.1021/acs.est.5b00006

[12] I.M. Perez De Vargas Sansalvador, C.D. Fay, J. Cleary, A.M. Nightingale, M.C. Mowlem, , D. Diamond, Sensors and Actuators, B: Chemical 225, 369 (2016) doi: $10.1016 /$ j.snb.2015.11.057

[13] M. Schirrmann, R. Gebbers, E. Kramer, J. Seidel, Sensors 11, 573 (2011) doi: $10.3390 / \mathrm{s} 110100573$

[14] A. Mroz, M. Borchardt, C. Diekmann, K. Cammann, M. Knoll, C. Dumschat, Analyst 123, 1373 (1998) doi: 10.1039/a708992i

[15] P. Spitzer, B. Werner, Analytical and Bioanalytical Chemistry 374, 787 (2002) doi: 10.1007/s00216-002-1453-1

[16] Yi Hu, Shu Wang, Ming Zhu, MATEC Web of Conferences 59, 01006 (2016) doi: 10.1051/matecconf/20165901006

[17] Y.-S. Li, L.-C. Shi, X.-F. Gao, J.-G. Huang, Desalination 390, 62 (2016) doi: 10.1016/j.desal.2016.04.008 